\title{
NEWER MANAGEMENT TIPS FOR PHACOEMULSIFICATION OF BRUNESCENT NUCLEI
}

\author{
Abhishek Kumar1, Shilpi Agrawal2, Sharwan Kumar Kedia ${ }^{3}$, Ashish Kumar 4 , Prasansha Narnoli ${ }^{5}$ \\ ${ }^{1}$ Senior Resident, Department of Ophthalmology, Lord Buddh Koshi Medical College \& Hospital, Saharsa, Bihar. \\ ${ }^{2}$ Consultant, BILAS Netralaya, Patna. \\ ${ }^{3}$ Consultant, Laxmi Netralaya, Arrah. \\ ${ }^{4}$ Consultant, Aravind Eye Hospital, Madurai. \\ 5Junior Resident, Department of Ophthalmology, Himalayan Institute of Medical Sciences, Dehradun.
}

ABSTRACT

\section{BACKGROUND}

Phacoemulsification is the standard technique for elective cataract surgery worldwide. In spite of recent advances in techniques and technology, the management of black and brunescent cataracts remains a challenge for even experienced surgeons.

Objective- Successful management tips that surgeons need to understand first and then employ it with specific strategies to achieve gratifying result in these cases.

\section{DISCUSSION}

Complete nuclear division of a rock-hard cataract can be very difficult using conventional techniques. Complete division may be achieved by starting the fracture at the posterior portion of the nucleus and keeping in mind the various challenges these brunescent nuclei present while chopping.

\section{CONCLUSION}

With a judicious mix of proper technique and appropriate technology including power modulation and enhanced fluidics, best results can be achieved in these cases.

\section{KEYWORDS}

Phacoemulsification, Brunescent Nuclei, Chopping.

HOW TO CITE THIS ARTICLE: Kumar A, Agrawal S, Kedia SK, et al. Newer management tips for phacoemulsification of brunescent nuclei. J. Evolution Med. Dent. Sci. 2017;6(53):4048-4051, DOI: 10.14260/Jemds/2017/875

\begin{abstract}
BACKGROUND
Phacoemulsification has gained acceptance since its introduction in the 1960s and has become the standard technique for elective cataract surgery worldwide. ${ }^{1,2,3}$ In spite of recent advances in techniques and technology, the management of black and brunescent cataracts remains a challenge for even experienced surgeons. The absence of a protective epinuclear layer, the paucity of cortex, the fragility of the capsule, and the laxity of the zonules all increase the risk of injury to the supportive structures of the lens during surgery. Longer phaco times at higher energy levels, increase not only the risk of corneal endothelial damage but is also characterised by certain problems, such as corneal oedema, capsule-zonular complications and wound burn. $2,3,4,5,6,7,8$

Many techniques to effectively remove hard nuclei and therefore minimise surgical damage have been developed in recent years. Stop-and-chop and phaco chop have become popular approaches, although in certain cases, performing safe and effective phacoemulsification with the conventional chopping technique remains a daunting task for the reasons described above. $1,2,4,7,8,9,10$
\end{abstract}

Financial or Other, Competing Interest: None.

Submission 29-05-2017, Peer Review 23-06-2017,

Acceptance 28-06-2017, Published 03-07-2017.

Corresponding Author:

Dr. Abhishek Kumar,

Senior Resident,

Lord Buddh Koshi Medical College \& Hospital,

Nh-107, Baijnathpur,

Saharsa-852201, Bihar.

E-mail: kediaabhi@gmail.com

DOI: $10.14260 /$ jemds $/ 2017 / 875$

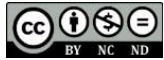

Successful management requires that surgeons first understand and then employ specific strategies to achieve gratifying result in these cases. Consider a scleral incision once in a while, no matter how skilled you may be as a phaco surgeon or how good the equipment may be, you will encounter a nucleus that is simply too dense to be emulsified. Or you may sense at a certain moment that things are about to "go south," a sudden deepening of the anterior chamber, for instance, or undue difficulty in manoeuvring the nucleus. When these things happen, the smart move is to bail and convert to ECCE. For this reason, it's a prudent move to perform a scleral tunnel incision in cases with extraordinarily dense nuclei, just in case the need to convert arises. ${ }^{11}$

\section{DISCUSSION}

Complete nuclear division of a rock-hard cataract can be very difficult using conventional techniques because the radial suture plane of these lenses tends to have a strong adhesive quality around the posterior epinucleus, forming a dense posterior nuclear plate.2,3,7 Chopping techniques at the anterior or equatorial portion can lead to incomplete fracture because deeper layers of the lens keep the nuclear fragments attached to each other, and partial fragmentation may result in unwanted outcomes during the procedure. ${ }^{2}$ One usually tries to complete the division by moving the instruments and the fragments outward to extend the centrifugal traction, but this may inadvertently lead to excessive capsular bag distortion and stress to the zonular fibres. The above complications can be avoided and a complete division achieved by starting the fracture at the posterior portion of the nucleus, ensuring that no fibres remain attached. 


\section{Multiple Challenges of the Brunescent Nucleus}

Virtually every step of cataract surgery is more difficult in the setting of a mature, brunescent nucleus. The absence of a good red reflex certainly poses problems to the capsulorhexis step. However, without a red reflex, even a successfully completed capsulorhexis remains difficult to see during nuclear emulsification, which increases the risk of damaging the edge with the chopper or phaco tip.

\section{Other Challenges to Consider \\ Capsular-lenticular Block}

During hydrodissection, an elevating hard nucleus is more likely to seal the capsulorhexis from below. Because fluid cannot escape the capsular bag, any additional fluid injected at this point of time will distend and eventually rupture the posterior capsule. The surgeon may not recognise this problem until the nucleus suddenly descends in to vitreous through the torn posterior capsule following the first sculpting stroke.

\section{Incisional Burns}

If the overlying viscoelastic is not initially aspirated away, it can mix together with the hard nuclear emulsion to clog the phaco tip. In the absence of any fluid exit, there is no gravityfed irrigation inflow, and a burn immediately develops as ultrasound commences. Particularly with a clear corneal incision, moderate stromal whitening and heat shrinkage can result from the higher levels of continuous phaco power. It is the combination of longer phaco time and greater phaco needle stroke length that generates excessive heat compared to routine cases.

\section{Endothelial Cell Loss}

As evidenced by increased corneal oedema on the first postoperative day, endothelial cell loss is much greater with hard nuclei. The increased density and volume of nuclear material necessitates greater ultrasound power and time. Because of the increased stroke length, and because rigid nuclear fragments do not mould as well to the phaco tip, there is poor followability and much greater chatter occurring at the phaco tip. This produces excessive particulate turbulence within the anterior chamber, which traumatises the endothelium.

\section{Posterior Capsule Rupture}

There is proportionally more endonucleus and less epinucleus with hard cataracts, which can double the volume of solid material requiring emulsification. This also forces the phaco tip to work much closer to the peripheral and posterior capsule, particularly when sculpting a deep central trough. Unlike a softer nucleus, which absorbs instrument pressure like a pillow, a dense nucleus is as rigid as a wooden board. As such, it more directly transmits all of the instrumentation forces directly to the posterior or peripheral capsule.

\section{Weak Zonules}

Very hard nuclei are often associated with weak zonules. Weak zonules are particularly problematic if there is little or no epinucleus present. Normally, the soft epinuclear shell restrains a lax posterior capsule from trampolining toward the phaco tip as the last nuclear fragment is removed. Without the epinucleus, the exposed posterior capsule is more likely to billow toward the phaco tip with even the slightest degree of post-occlusion surge. The sharp edges of the brunescent fragments and the greater capsular laxity caused by weak zonules further increase the risk of capsular rupture. If one reacts defensively by emulsifying the last fragment closer to the cornea, then one further heightens the risk of endothelial cell loss.

\section{Preoperative Evaluation}

A meticulous preoperative evaluation is required prior to scheduling phacoemulsification. Because there is potential for collateral intraocular damage with hard nuclei, one should evaluate the ocular tissues including the cornea. If facilities are available, perform specular microscopy and pachymetry to objectively evaluate the corneal status. In rare cases, consider manual small-incision cataract surgery (SICS) over phacoemulsification in the presence of an unhealthy cornea. Extracapsular cataract extraction is another alternative in these situations. Other parameters that should routinely be evaluated during the preoperative examination include anterior chamber depth, maximum mydriasis, and presence of iridophacodonesis. We should always grade the hardness of the nucleus at the slit lamp.

When the degree of cataract precludes a good view of the fundus, it is wise to take a B-scan ultrasonograph of the posterior segment to detect vitreous opacities or retinal detachment. Because optical biometry may not be the best strategy to calculate IOL power in these super-hard cataracts, it is better to resort to immersion A-scan biometry. ${ }^{12}$

\section{Pearls and Strategies for the Brunescent Nucleus}

1. Capsular Dye- Trypan blue 0.06 percent should be the agent of choice for the mature nuclear cataract. Trypan blue provides a more intense and persistent colouration, which enhances visibility of the capsulorhexis edge during emulsification. ${ }^{13}$

2. Capsulorhexis- An intact, relatively large capsulorhexis is of paramount importance for hard nuclei. Many elderly patients have a lustreless cornea or a thick arcus senilis that impairs visibility. A visible rhexis margin can prevent inadvertent damage during nuclear emulsification, such as phacoemulsification of the anterior capsular edge or passage of the chopper on top of the anterior capsule. If intralenticular pressure is high, it is better to perform a two-stage procedure, starting with a small capsulorhexis of 3.0 to $3.5 \mathrm{~mm}$ in diameter to reduce the risk of rhexis run-off to the periphery. After that the rhexis can be enlarged to the desired size by decompressing the capsular bag with aspiration of flocculent cortex. ${ }^{12}$

3. Avoid Capsular Block during Hydrodissection- To avoid capsular-lenticular block, the hydrodissection should be terminated as soon as the solid nucleus elevates against the capsulorhexis. One must resist the temptation to continue injecting until the migrating posterior fluid wave completely crosses behind the nucleus. Instead, tap the centre of the elevated nucleus to dislodge it posteriorly before resuming hydrodissection from the opposite quadrant. A right-angled hydrodissection cannula facilitates the latter step. 
4. High Vacuum and Burst Mode- While advantageous under ordinary circumstances, these phacodynamic parameters become particularly important in managing the dense cataract. Burst mode and high vacuum combine to provide a maximal grip. Inadequate holding power makes it more likely that the vertical chopper will dislodge pieces from the phaco tip, and makes it more difficult to elevate large fragments out of the bag. Particularly with a brunescent lens, continuous mode cavitation cores out the firm material surrounding the phaco tip. This facilitates sculpting but prevents total tip occlusion by eroding the seal. Because high vacuum levels require a well-occluded phaco tip, burst mode is particularly advantageous for the dense lens.

5. Sculpt a Central Pit Prior to Diagonal Phaco Chop- By reducing phaco power and time as well as stress on the zonules, chopping is a superior phaco technique for the hard nucleus. In the absence of an epinuclear shell, horizontal phaco chop is contraindicated, and vertical chop also is able to transect the leathery posterior plate better. Sculpting a deep pit in the central anterior nucleus allows the surgeon to penetrate the bulky nucleus more deeply and peripherally with the phaco tip than if the nucleus was impaled without this step. Louis D. Nichamin, MD, has named this strategy crater-quick chop. To better impale the nucleus, one should maximally retract the irrigation sleeve and bury the tip to the hilt. A very sharp vertical chopper will best incise into the dense nuclear face without displacing it. One can place the chopper peripherally beneath the blue-stained anterior capsule, and then chop in a diagonal direction toward the buried phaco tip. This adds a slight horizontal vector force that compresses the fragment against the phaco tip during the vertical chop.

6. Subchop brunescent Fragments- Regardless of whether the initial nuclear fragmentation is accomplished by divide-and-conquer, stop-and-chop or pure chopping methods, the resulting fragments are much larger and denser than those encountered with typical nuclei. One can employ horizontal chopping to subdivide these large fragments into smaller, bite-sized pieces. This reduces the tendency for oversized fragments to deflect away from the vibrating phaco tip. By reducing particle chatter and turbulence within the anterior chamber, endothelial cell loss can be lessened.

7. Hyperpulse Technology-Originally introduced as WhiteStar technology with the AMO Sovereign, hyperpulse power modulation is now available from a number of companies. The widely recognised advantages of hyperpulse are a dramatic reduction in heat and energy production, and improved followability because of decreased chatter of fragments at the phaco tip. The combination of subchopping and hyperpulse mode can significantly reduce particle turbulence within the anterior chamber, this probably is the most important factor in decreasing endothelial cell loss with brunescent cataracts.
8. Maximally Retentive Viscoelastics-With the prolonged operative times associated with hard nuclei, viscoelastic washout is a far greater problem than with most typical cases. Dispersive viscoelastics, such as Viscoat and Vitrax, or the viscoadaptive Healon 5, if utilised properly, are able to coat and protect the endothelium for longer periods than their cohesive counterparts. As all viscoelastics eventually wash away, consider stopping to replenish the protective endothelial layer midway through the nuclear removal step.

9. Viscoelastic Vault- Roger F. Steinert, MD, has coined this term to describe a technique for deploying a dispersive viscoelastic as an artificial epinucleus for the brunescent nucleus. In cases where the epinucleus is scant or absent, one should temporarily stop phaco before removing the last remaining fragments. A generous amount of dispersive viscoelastic is injected in front of and behind the fragments in order to partially fill the capsular bag. Because dispersive viscoelastics better resist aspiration by the phaco tip, they will better restrain the lax and exposed posterior capsule from trampolining toward the phaco tip. This viscoelastic layer also cushions the capsule from being poked by the brunescent fragments. With less concern about proximity to the posterior capsule, the surgeon is less likely to phaco the last fragment too close to the endothelium.

10. Watch carefully for "lost" chips- Very dense nuclei often break into many tiny particles that can get trapped in the eye during the phaco procedure. Care must be taken to look for and remove all the little chips that may be hiding in the anterior chamber, in the incision, or in the iris. It's wise to re-enter the phaco incision with the phaco tip and to irrigate the side port(s) at the end of the case to free and aspirate chips that may be trapped in the incisions. ${ }^{11}$

11. Capsular Tension Rings and Capsule RetractorsCapsule retractors can stabilise a wobbly nucleus with weak zonules during phaco. Their insertion is again dependent upon effective staining and capsulorhexis visualisation. CTRs impede cortical cleanup, and they don't provide the anterior-posterior support or torsional stability for the bag that the capsule retractors do. However, CTRs are certainly invaluable for stabilising a moderately weakened capsular bag for IOL implantation and longer-term centration. ${ }^{13}$

\section{CONCLUSION}

Despite these helpful devices and strategies, individual surgeons must assess their personal limit in terms of how hard a lens they can safely emulsify. It is far better to accept the larger incision of a Small-Incision Cataract Surgery or standard extracapsular cataract extraction than to have a dropped nucleus or decompensated cornea. Even when proceeding with phaco, the surgeon must have a contingency plan for unexpected problems such as weak zonules or poor visibility. For the most challenging cases, consider administering a regional anaesthetic block and have no ego to convert to a SICS or standard ECCE. ${ }^{13}$ With a judicious mix of 
proper technique and appropriate technology including power modulation and enhanced fluidics, best results can be achieved in these cases. ${ }^{12}$

\section{REFERENCES}

[1] Vajpayee RB, Kumar A, Dada T, et al. Phaco-chop versus stop-and-chop nucleotomy for phacoemulsification. J Cataract Refract Surg 2000;26(11):1638-41.

[2] Kim HK. Decrease and conquer: phacoemulsification technique for hard nucleus cataracts. J Cataract Refract Surg 2009;35(10):1665-70.

[3] Hwang HS, Kim EC, Kim MS. Drill-and-crack technique for nuclear disassembly of hard nucleus. J Cataract Refract Surg 2010;36(10):1627-30.

[4] Singh R., Vasavada AR, Janaswamy G. Phacoemulsification of brunescent and black cataracts. J Cataract Refract Surg 2001;27(11):1762-9.

[5] Gimbel HV. Principles of nuclear phacoemulsification. In: Steinert RF. edr. Cataract surgery, techniques, complications and management. $2^{\text {nd }}$ edn. Philadelphia, PA: Saunders 2004:153-81.

[6] Pereira ACA, Porfírio F, Freitas LL, et al. Ultrasound energy and endothelial cell loss with stop-and-chop and nuclear preslice phacoemulsification. J Cataract Refract Surg 2006;32(10):1661-6.
[7] Kamoi K, Mochizuki M. Phaco forward-chop technique for managing posterior nuclear plate of hard cataract. J Cataract Refract Surg 2010;36(1):9-12.

[8] Vasavada AR, Raj M. Multilevel chop technique. J Cataract Refract Surg 2011;37(12):2092-4.

[9] Gimbel HV. Divide and conquer nucleofractis phacoemulsification: development and variations. J Cataract Refract Surg 1991;17(3):281-91.

[10] Koch PS, Katzen LE. Stop and chop phacoemulsification. J Cataract Refract Surg 1994; 20(5): 566-70.

[11] Colvard DM. Complicated cataract cases Phacoemulsification of the rock hard cataract. https://www.eyeworld.org/article-

Phacoemulsification-of-the-rock-hard April 2012.

[12] Chakarbarti A. Common-phaco-techniques-for-rockhard-nuclei/ cataract \& refractive surgery today. crstodayeurope.com/articles/2013.

[13] Chang DF. Ten strategies for the rock-hard nucleus. Eye Net 2005.

http://www.aao.org/publications/eyenet/200503/pe arls.cfm 